\title{
Nanduty
}

ISSN:2317-8590

\section{EL SUICIDIO COMO PROBLEMA GUBERNAMENTAL EN LA CIUDAD DE CÓRDOBA, ARGENTINA.}

\author{
Santiago Manuel Romero (UNC-IDH/CONICET) - \\ santiagom.romero@gmail.com
}

\begin{abstract}
RESUMEN
En este trabajo me propongo indagar respecto de la producción social del suicidio como problema gubernamental en la ciudad de Córdoba/Argentina de los últimos años. Para ello analizaré etnográficamente un conjunto de prácticas y saberes en pos de la evitación del suicidio por parte de una ONG local. También me detendré en algunos análisis clásicos sobre el suicidio considerando que participaron de la producción social del suicidio como un problema gubernamental. La pregunta que orientará el trabajo será respecto de las formas de gobierno sobre la vida en la córdoba contemporánea, considerando que participan de un universo de prácticas gubernamentales que toman formas particular en contextos neoliberales.
\end{abstract}

\section{PALABRAS CLAVE: SUICIDIO, GUBERNAMENTALIDAD, ETNOGRAFÍA}

\section{RESUMO}

Neste artigo, proponho investigar a produção social do suicídio como um problema governamental na cidade de Córdoba/Argentina nos últimos anos. Para este fim, analisarei etnograficamente um conjunto de práticas e conhecimentos em busca da prevenção do suicídio por uma ONG local. Também me debruçarei sobre algumas análises clássicas do suicídio, considerando que eles participaram da produção social do suicídio como um problema governamental. A questão que orientará o trabalho será sobre as formas de governo sobre a vida em córdoba contemporânea, considerando que elas participam de um universo de práticas governamentais que tomam formas particulares em contextos neoliberais.

\section{PALAVRAS-CHAVE: SUICÍDIO, GOVERNO, ETNOGRAFIA}

\begin{abstract}
In this paper I propose to investigate the social production of suicide as a government problem in the city of Córdoba/Argentina in recent years. To this end, i will ethnographically analyze a set of practices and knowledge in pursuit of suicide avoidance by a local NGO. I will also dwell on some classical analyses of suicide considering that they participated in the social production of suicide as a governmental problem. The question that will guide the work will be regarding the forms of government about life in contemporary cordoba, considering that they participate in a universe of governmental practices that take particular forms in neoliberal contexts.
\end{abstract}

KEYWORDS: SUICIDE, GOVERNMENTALITY, ETHNOGRAPHY 


\section{Nanduty}

ISSN:2317-8590

\section{INTRODUCCIÓN}

En este trabajo me propongo indagar respecto de la producción social del suicidio como problema gubernamental en la Ciudad de Córdoba de los últimos años. Estas indagaciones formaron parte de la investigación etnográfica que desarrolle entre 2015 y 2018 como Trabajo Final de la Licenciatura en Antropología en la Universidad Nacional de Córdoba. En esa pesquisa me propuse realizar un análisis etnográfico sobre un universo de prácticas, técnicas, saberes, discursos que se accionaban en la Ciudad de Córdoba para administrar un fenómeno que se configuraba como un "problema social" (Lenoir 1993). ${ }^{1}$ El objeto de investigación que orientó mi trabajo fue formulado respecto de las acciones de gestión tendidas en torno del suicidio, por parte de un Centro de Asistencia al Suicida (CAS) en la ciudad de Córdoba, con el fin de asistir a personas en crisis y de prevenir situaciones de riesgo. ${ }^{2}$

La pesquisa que desarrollé implicó un abordaje etnográfico de técnicas de administración y gestión sobre poblaciones locales, en especial de segmentos juveniles, accionadas por las voluntarias del CAS, entendiendo que forman parte de mecanismos más extensos de gestión de poblaciones y de vidas. Estas preguntas de investigación tuvieron como inspiración las propuestas de Michel Foucault (1988) en relación al análisis microfísico del poder, cuya apuesta radica en estudiar el cómo del poder

$1 \quad$ El trabajo se titula: La vida puede más. Formas de asistencia y prevención de un Centro de Asistencia al Suicida de la Ciudad de Córdoba, Argentina. Contó con la dirección de María Gabriela Lugones y María Lucía Tamagnini. La vida puede más, era una frase que aparecía en la folletería del CAS.

2 Emplearé las itálicas para voces y expresiones de las voluntarias así como para documentos y materiales empleados por ellas en su labor o que tratan sobre el suicidio y su formas de evitación. Cuando cite fragmentos de entrevistas, aparecerán en párrafo aparte y con mayores márgenes. Con las "comillas dobles" marcaré categorías analíticas y fragmentos de literatura de poca extensión. Los fragmentos de más de tres renglones aparecerán en párrafo aparte. Las 'comillas simples' serán empleadas para señalar alguna categoría cuyo significación esta puesta en duda o relativizada.

Los nombres empleados para nominar a las voluntarias del CAS son ficticios. 


\section{Nanduty}

ISSN:2317-8590

partiendo de sus ejercicios, mecanismos y efectos en niveles capilares. Esta forma de concebir el poder supone considerarlo como una acción, no como un capital.

Tomé como objeto analítico las específicas formas de ejercicio de poder que se articulaban en la asistencia y prevención del suicidio, focalizando en las efectuadas por las voluntarias del CAS. ${ }^{3}$ Resultó clave la conceptualización que Foucault propone de "gobierno", para quien serían acciones que se realizan sobre acciones posibles, lo cual implica una relación entre sujetos actuantes o susceptibles de actuar. Los actos de gobierno inciden sobre las posibilidades de acción de las conductas de los sujetos sobre los que actúa. Estas acciones sólo en última instancia obligan o impiden, más bien se caracterizan por su carácter positivo, esto es: las acciones de gobierno incitan, seducen, inducen, facilitan o dificultan (Foucault 1988: 15). Para Foucault, las técnicas y tácticas de gobierno son difusas y ejercidas por una multiplicidad de agentes, no sólo por el estado (Foucault 1988: 9). En esta dirección, consideré que podían ser aprehendidas las técnicas de gobierno accionadas por las voluntarias del CAS como integrando un horizonte de estrategias de gubernamentalidad ejercidas en la Córdoba contemporánea.

Si la nueva modalidad del poder descrita por Foucault (2013) tiene por característica el "hacer vivir y dejar morir", una investigación sobre prácticas orientadas a la evitación del suicidio parecería ser una oportunidad potable para reflexionar sobre los esfuerzos por mantener a los individuos "dentro" de esa esfera de competencia. En esos términos, el suicidio parecería, y así lo ha dejado delineado el mismo Foucault

3 El voluntariado en el CAS, implicaba el desarrollo de actividades ad honorem, para las cuales recibían una formación específica. Emplearé la voz "voluntaria" para referirme a aquellas personas que participaban del voluntariado del CAS. Mantendré el singular y el plural en femenino para recalcar el hecho de que, en su mayoría, mis interlocutoras se identificaban como mujeres, mientras que la proporción de varones era menor. No me detendré en la cuestión de la distribución diferencial de las tareas de cuidado marcada por el género dado que es un tópico largamente desarrollado (Esquivel, Faur \& Jelin 2009; Pautassi \& Zibecchi 2013; Zibecchi 2014; Pedroso de Lima 2016), pero está en el horizonte de las problemáticas tratadas en la investigación. 


\section{Nanduty}

ISSN:2317-8590

(2010), una forma de libertad respecto de esas formas de poder. Sin embargo, en el trabajo de campo etnográfico, pude apreciar cómo el énfasis era puesto en la vida y en las formas en que la vida podía más. De modo que, más que evitar un tipo de muerte, las actuaciones de las voluntarias se orientaban a una "gestión de las emociones", se orientaban a contribuir a una tramitación pacificada de crisis emocionales. Me propondré con este trabajo una abordaje múltiple del suicidio como fenómeno. Primero visitando de una forma bastante interesada la obra clásica de Durkheim sobre el suicidio, y a continuación presentando cómo lo abordaban las voluntarias del CAS. Todo esto con el objetivo de proporcionar carnatura etnográfica a los saberes respecto de las formas de ejercicio de poder sobre la vida, en este caso, respecto a la producción del suicidio como un problema gubernamental y de técnicas orientadas a la gestión emocional.

\section{DEL SUICIDIO COMO PROBLEMA GUBERNAMENTAL}

El suicidio fue producido como objeto de preocupación de las ciencias sociales desde sus momentos de consolidación. Parecería inevitable mencionar la obra de Emile Durkheim, "El Suicidio" (1998[1897]), como prolegómeno de las investigaciones respecto de ese fenómeno. El movimiento analítico realizado por Thomas Tierney en su artículo "The governmentality of suicide: Peuchet, Marx, Durkheim, and Foucault" (2010) permitió realizar una lectura tangencial de esta obra. ${ }^{4}$ Particularmente pues el análisis encarado por Tierney sobre la obra de Durkheim, y por extensión, sobre las preocupaciones de la sociología del siglo XIX sobre el suicidio, se hilvanaron fuertemente con las preocupaciones analíticas que venía desarrollando en mi pesquisa.

La investigación de Durkheim en "El Suicidio" generalmente es presentada como 


\section{Nanduty}

ISSN:2317-8590

una obra fundante de la sociología y en los análisis desde las ciencias humanas respecto de ese fenómeno. Durkheim, luego de la publicación de "Las reglas del método sociológico" (1985), elige encarar su reconocida investigación sobre las tasas de suicidio en Francia que consagraría la metodología propuesta para la incipiente disciplina. Al construir como objeto analítico las "tasas de suicidio" intenta señalar cómo éstas constituían hechos sociales suis generis, y por lo tanto serían competencia de la sociología. De esta forma disputaba ser la voz autorizada frente a otras disciplinas también en importante crecimiento como ser la Psicología y la Psiquiatría.

El artículo de Tierney, examina dos ejemplos de lo que llama la "fascinación de la sociología del siglo XIX con el suicidio" (Tierney 2010: 358; siguiendo a Giddens 1965; Edwards \& Osborne 2005) y cruza sus lecturas con el análisis histórico de Foucault respecto de la configuración de la "Gubernamentalidad" (2006 [1978]). Toma por un lado el texto mencionado de Durkheim y por el otro examina lo que llama un tratado proto-sociológico sobre el suicidio publicado en 1838, 50 años antes de la obra de Durkheim. Se trata de las memorias de un administrador policial francés, Jacques Peuchet, publicadas póstumamente en 1838 bajo el título, "Mémoires des archives de pólice de Paris, pour servir à l'histoire de la morale et de la pólice, depuis Louis XIV jusqu'à nos tours". La versión que analiza es la traducción realizada y publicada por Karl Marx de la sección titulada "Del suicidio y sus causas". Tierney plantea que si bien el suicidio no era la preocupación principal de Peuchet o de Marx, resulta interesante su análisis dado que tanto el texto como la fascinación del joven Marx por él emergen en la tensión entre la policía y el liberalismo que Foucault identificó como clave para el surgimiento de la gubernamentalidad (Tierney 2010: 359).

En el contexto de la historia de la gubernamentalidad que Foucault presentó en sus cursos de finales de los años 70, la fascinación sociológica del siglo XIV por el suicidio aparece como parte del establecimiento de una nueva forma de 


\section{Nanduty}

ISSN:2317-8590

racionalidad política que gobierne la conducta de individuos basada en los patrones y regularidades develadas por el análisis estadístico de la población. Como hemos visto, esta racionalidad gubernamental emergió de la fértil tensión entre la perspectiva mercantilista estado-céntrica, la cual dio origen a la policía, y la perspectiva liberal de la sociedad civil, que produjo las ciencias humanas. (Tierney 2010: 383. La traducción me pertenece) ${ }^{5}$

En el panorama planteado por Foucault, cuando la "policía" reduce sus funciones a las 'represivas' o 'negativas' que hoy conocemos, otras disciplinas se encargarán de desarrollar las funciones 'positivas'. Recordemos que la antigua policía analizada por Foucault refería a los medios necesarios para fortalecer un Estado desde el interior, era un "arte de gobierno" tendiente a analizar y potenciar la vida de individuos que viven en un territorio (Foucault 1996; 2006 [1978]). Cuando se produce aquella transformación entre los siglos XVIII y XIX serán disciplinas como la economía política, la psicología y la sociología las que se ocupen de la dimensión 'positiva' de la policía.

Sabemos cuán central será la estadística como técnica clave en la producción de saberes por parte de la sociología. Saberes con los cuales la sociedad civil podía ser gestionada y administrada. En ese panorama, podemos pensar que el intento de Durkheim de establecer la sociología como único saber respecto del suicidio era un modo de instaurar la disciplina como una ciencia crucial para la administración gubernamental. Así es que Tierney entiende que "El suicidio" de Durkheim no sólo es un clásico de la sociología, sino también un clásico de la gubernamentalidad.

Al considerarlas con Tierney como investigaciones con cierto interés gubernamental, me permitieron pensar cómo las ciencias humanas participaron de la

5 "In the context of the history of governmentality that Foucault presented in his courses from the late 1970s, the nineteenth century sociological fascination with suicide appears as part of the establishment of a new form of political rationality that governs the conduct of individuals based upon the patterns and regularities revealed by statistical analyses of the population. As we have seen, this governmental rationality emerged out of a fertile tension between the state-centered mercantilist perspective, which gave rise to the police, and the liberal perspective on civil society, which produced the human sciences." 


\section{Nanduty}

ISSN:2317-8590

configuración del suicidio ya no necesariamente como un problema jurídico o religioso sino como un problema gubernamental, produciendo saberes eficaces para su gestión. Con la esperanza de no quedar entrampado en una preocupación gubernamental, orienté mis esfuerzos analíticos a pesquisar no el fenómeno del suicidio en Córdoba, con sus cifras, modalidades o "tasas", sino las específicas formas en las que se lo evitaba o prevenía. Modalidades que podrían pensarse conformando una tradición específica de gestión.

\section{EL CENTRO DE ASISTENCIA AL SUICIDA DE LA CIUDAD DE CÓRDOBA}

El Centro de Asistencia al Suicida y la Persona en Crisis (CAS) de la ciudad de Córdoba es una asociación civil conformado por un grupo de voluntarias cuyas actuaciones se orientan a la evitación del suicidio desde mediados de los años 80'. Las labores de las voluntarias del CAS se organizaban en dos tipos de actividad. La primera e históricamente desarrollada por la asociación civil era un servicio de contención telefónica, para el cual contaban con dos líneas telefónicas. En los llamados, las voluntarias tentaban ayudar a los consultantes que marcaban sus números a tramitar crisis emocionales. Ya en los últimos 15 años venían desarrollando talleres de prevención, que se realizaban en escuelas del nivel medio de la ciudad y estaban orientados específicamente a poblaciones de jóvenes y adolescentes. ${ }^{6}$

Considerando que mi interés estaba centrado en la tradición administrativa

6 Desarrollé un trabajo de campo etnográfico entre los años 2014 y 2016. Durante los primeros meses realicé una primera instancia exploratoria; luego, entre abril y mayo de 2015 formalicé un vínculo con las voluntarias y en junio de ese año comencé a participar de las reuniones mensuales del equipo de prevención, luego del curso de formación para nuevas voluntarias telefónicas y a los talleres de prevención en escuelas del nivel medio de la ciudad de Córdoba. Tareas que se extendieron hasta diciembre de 2016, al tiempo que se llevé a cabo entrevistas con varias de las voluntarias. 


\section{Nanduty}

ISSN:2317-8590

cordobesa respecto de la evitación del suicidio, me pareció importante retomar el relato de la constitución del CAS. Según me relatara una de las voluntarias, el CAS se conformó a partir de un grupo de personas conectadas por la pertenencia propia, o de sus cónyuges, a la Fuerza Aérea. ${ }^{7}$ Catalina Astorga de Romero, cuyo esposo ocupaba un alto cargo en dicha fuerza armada, conoce las actividades del Centro de Asistencia al Suicida de la Ciudad de Buenos Aires mientras visita a su hermana. A partir de dicho contacto comienza a reunir un grupo inicial para conformar lo que será el CAS Córdoba. Entre el 1ro de julio de 1984 y el 29 de julio de 1985 se formó la primera comisión directiva y las voluntarias realizaron una formación siguiendo la forma de trabajo del CAS Buenos Aires. ${ }^{8}$ El CAS tomó el formato de una asociación civil, que cuenta con una organización establecida bajo los requerimientos de la Dirección de Inspección de Personas Jurídicas del ejecutivo provincial. La comisión directiva, durante varios años, se compuso por ese grupo de personas conocidas por relaciones con la Fuerza Aérea. Sus funciones principales eran la presidencia y administración del CAS, mientras que los operarios telefónicos se encargaban de la atención telefónica. ${ }^{9}$

Otra de las figuras fundantes en la historia del CAS, fue Clyde Capolongo de

7 La Fuerza Aérea cuenta con una importante comunidad en la ciudad de Córdoba, donde están instaladas el Área Logística Córdoba, la Escuela de Aviación Militar, el Instituto Universitario Aeronáutico, la Escuela de Suboficiales de la Fuerza Aérea. Asimismo se encontraba la antigua Fábrica Militar de Aviones, actualmente FadeA, Fábrica Argentina de Aviones.

8 Si bien existieron y existen varios CAS en distintas ciudades y provincias del país, y otras organizaciones similares, no logré acceder a literatura que abordase etnográficamente o sociológicamente sus labores. Cuando ya había comenzado a escribir mi trabajo final la antropóloga Isis Ribeiro Martins publicó su tesis doctoral titulada "A linha da vida": escuta e gestão do sofrimento em serviços telefônicos de apoio emocional" dirigida por Adriana Vianna en el PPGAS-MN de la Universidad Federal de Rio de Janeiro, Brasil. La tesis, defendida en 2015 y publicada en 2017, aborda las prácticas de apoyo emocional de un Centro de Valorización de la Vida (CVV) que, en una escala mayor y articulada a nivel federal, realiza tareas en pos de la evitación del suicidio.

$9 \quad$ Actualmente se rige según establece el Código Civil y Comercial de la Nación en su artículo 171 y la Resolución N ${ }^{\circ}$ 70/2009 de la Dirección General de Inspección de Personas Jurídicas de la provincia. 


\section{Nanduty}

ISSN:2317-8590

Herreros, psiquiatra, ${ }^{10}$ quien junto a Juana Grangeat, psicóloga, se encargaron de la formación de las primeras voluntarias y de la supervisiones de los llamados. La primera convocatoria tuvo una concurrencia de alrededor de 150 personas, que dieron lugar al primer grupo de 20 voluntarios. Vemos, respecto del caso brasileño relatado por Martins (2016), una conformación laica y tempranamente ligada a los saberes PSI. Martins señalaba que el CVV en su origen estaba ligada al cristianismo, tanto en la 'vocación' de sus voluntarios como en los saberes fundantes del servicio. Si bien en el CAS reconocían que los primeros servicios de este tipo estuvieron ligados al cristianismo, este factor no era invocado en los relatos de las voluntarias. Mientras que sí lo eran las enseñanzas que les habían dejados los profesionales que habían participado y/o dirigido la institución. Frente a este relato laico y vinculado al mundo PSI (aunque no participando de él directamente), ${ }^{11}$ Ribeiro relata cómo fue un momento de disrupción en la historia institucional el momento de laicización del CVV.

Por su parte, el programa de prevención del CAS, comenzó a gestarse alrededor del año 2002, cuando las voluntarias empezaron a detectar que los jóvenes no hacían uso del servicio telefónico al mismo tiempo que las cifras de suicidio en ese rango etario comenzaban a incrementarse. Esta detección también estaba presente en la bibliografía que especialistas de la psicología, sociología y administradores estatales comenzaron a producir localmente un par de años antes, construyendo a las prácticas autodestructivas como una problemática en crecimiento.

10 Formaba parte del servicio de Psicología y Psiquiatría del Hospital Privado, un importante nosocomio de la ciudad de Córdoba.

11 Pese a esta cercanía con saberes PSI, las voluntarias se encargaban de señalar que la atención telefónica no era equivalente a una sesión de terapia y por muchos años no permitieron que profesionales de la psicología o psiquiatría participaran como operarias telefónicas. Esta opción fué menguando en el momento que realicé mi trabajo de campo, pues muchos estudiantes o graduados recientes de la carrera de Psicología se acercaron a realizar el voluntariado del CAS. Respecto de eso, es interesante que para ellos también implicaba un espacio de formación, pues reclamaron varias veces que en su carrera no había espacios curriculares específicos respecto de conductas suicidas y su evitación. 


\section{Nanduty}

ISSN:2317-8590

Casi una década de estudios y publicaciones específicas acerca de conductas autodestructivas entre jóvenes y adolescentes sirvieron en su momento como base para el trabajo preventivo de las voluntarias del CAS. ${ }^{12}$ Una de las referentes del tema en el ámbito local es la psicóloga María Martina Casullo, cuyas investigaciones fueron retomadas al momento del diseño de los talleres. ${ }^{13}$ En 1994, publicó un artículo titulado: Comportamiento suicida adolescente. Epidemiología y prevención (Casullo 1994); más adelante un libro Adolescentes en riesgo (1998), luego Comportamientos suicidas en la adolescencia. Morir antes de la muerte (Casullo et al. 2000). Podemos ver que en la configuración del "problema social" del suicidio en el contexto local, los adolescentes aparecen como una "población" especialmente merecedora de intervención, transformándose en un grupo en riesgo debido a los muchos problemas que experimentan. Motivo por el cual las voluntarias diseñaron un dispositivo de intervención particular para ser desarrollado en escuelas del nivel medio.

Al momento de mi pesquisa, el CAS estaba conformado por alrededor de 15 voluntarias en actividad. Podían distinguirse dos grupos, las operarias telefónicas que se encargaban del servicio de contención, y el equipo de prevención. En el primer grupo estaban las voluntarias de más antigüedad en la institución y no había un perfil especifico, muchas de las cuales eran profesionales de diversas áreas retiradas o jubiladas. Algunas de ellas tenían mas de 20 años de trabajo en el CAS. En el equipo de

12 Generalmente en la literatura específica la adolescencia comprende el rango entre los 15 y los 19 años de edad, mientras que los jóvenes serían aquellos entre 20 y 25 años. Esta distinción suele aplicarse también en las estadísticas, aunque algunas unifican un gran grupo de entre 15 y 25 como "jóvenes".

13 María Martina Casullo (1940-2008), fue Licenciada en Psicología, por la Universidad Nacional de Buenos Aires y Doctora en Psicología por la Ohio State University. En ocasión de la formación de aspirantes para el equipo de prevención, Gabriela (psicóloga que participaba del equipo) señaló que algunas de las herramientas que empleaban provenían de la psicología positiva. La Psicología Positiva es una corriente que plantea un rechazo el privilegio dado al análisis y estudio de patologías, debilidades o carencias emocionales. Propone atender a las fortalezas, capacidades de los individuos que contribuyen a su bienestar psíquico, de ahí su carácter positivo (Casullo 2006b). 


\section{Nanduty}

ISSN:2317-8590

prevención, que orientaba sus actuaciones a jóvenes de escuelas secundarias, había una mayor presencia de profesionales de la psicología, algunas de ellas jóvenes recién recibidas. Veamos a continuación algunas facetas de cómo concebían y orientaban sus prácticas las voluntarias, al tiempo que explicaban las conductas autodestructivas. ${ }^{14}$ Muchos de estos saberes eran transmitidos durante el curso de formación de voluntarias que pude acompañar durante el año 2015. Eran los saberes considerados como teóricos por las voluntarias y que cimentaban el oficio de la prevención del suicidio y de la contención a personas en crisis.

\section{DEL SUICIDIO Y LA CRISIS}

Las voluntarias sostenían que lo que ellas hacían era hacer pensar, incidir en la modalidad de tramitación de las crisis de los consultantes por medio de un reordenamiento de sus ideas. Cuando le pregunté a Irma, una de las voluntarias, qué era el CAS, me dijo lo siguiente

Para mí el CAS es una forma de atender crisis emocionales muy rápida y muy sencilla. Y a lo mejor cualquiera que me escucha puede pensar: Mira cómo lo define. Y si, es muy... es lo que te decía ahí, es muy sencilla. Entonces, en el CAS nosotros lo que atendemos son crisis emocionales, de cualquier tipo. (Entrevista con Irma, 13 de junio de 2016) ${ }^{15}$

Podemos ver que eran las categorías de crisis, de situaciones de crisis, personas en crisis y no la de 'suicidas' la que orientaba las actuaciones de las voluntarias. Así, en mi pesquisa consideré cómo la producción relacional de esta categoría implicaba formas

14 Por una cuestión de espacio no me detendré a detallar las especificidades de las técnicas telefónicas y de los talleres de prevención orientados a jóvenes, pero fueron un insumo crucial para las reflexiones aquí vertidas, ver Romero (2018).

15 Irma tenía 74 años en el transcurso de la investigación. Ejerció la docencia en el país y en México, donde vivió con su familia durante la última dictadura militar. Una vez retornados al país se instalaron en la ciudad de Córdoba. En el año 1986 comenzó el voluntariado en el CAS, como operaria telefónica. Cuando se formó el equipo de prevención participó un tiempo, pero durante el tiempo que compartí con ellas, ya no lo hacía. 


\section{Nanduty}

ISSN:2317-8590

específicas de administración, describiendo cómo se producía esta categoría en las prácticas para administrarla. ${ }^{16}$

Lo que llamaban Teoría de la Crisis era transmitida en la formación de nuevas voluntarias, instancia donde desarrollé parte de mi trabajo de campo. Allí empleaban un documento donde se retoma la siguiente definición:

Slaikeu (1996) define una crisis como "un estado temporal de trastorno y desorganización, caracterizado principalmente por una incapacidad del individuo para manejar situaciones particulares utilizando métodos acostumbrados para la solución de problemas y por el potencial para obtener un resultado radicalmente positivo o negativo." Se caracteriza por una gran desorganización emocional, perturbación y un colapso en las estrategias previas de enfrentamiento.

Existen muchas formas de clasificar las crisis. La más común incluye dos categorías: crisis evolutivas y crisis circunstanciales. (Documento Crisis-clase.)

Karl Slaikeu es psicólogo, mediador y experto en negociación radicado en Texas, U.S.A.. Además de tener trabajos en relación a la Intervención en Crisis (1996), se especializa en la resolución de conflictos en ámbitos organizacionales y también se desempeñó como asesor de las Fuerzas Armadas de U.S.A. en la misma temática. ${ }^{17}$ La crisis era entendida por las voluntarias como un estado que todos habíamos atravesado alguna vez, como algo normal en la vida de las personas. Era, de algún modo, un fenómeno universalizable que se caracterizaba por una desorganización emocional frente a la cual los sujetos no encontrarían fácilmente estrategias para la resolución airosa de tal estado. Las crisis evolutivas referían a las que se asociaban con distintas instancias vitales, como embarazos, nacimientos, muertes, paso a la adolescencia, a la

16 En "El mal que se adivina”, Adriana Vianna (1997), donde analiza el acciones policiales en relaciones a 'menores', realiza un interpelante movimiento analítico: en lugar de organizar su trabajo en torno de la noción de menor, centró su análisis en cómo tal personaje era creado. Así, su preocupación analítica se centró en la "relación de minoridad", resaltando el carácter relacional de la producción de categorías sociales y su vinculación con prácticas administrativas.

17 (http://karlslaikeu.com/about-karl-slaikeu/ Última visita 26/11/2017) 


\section{Nanduty}

ISSN:2317-8590

adultez, jubilación, entre otras. Por su parte, las crisis circunstanciales tenían que ver con eventos de la más diversa orden que podían desencadenar un estado de crisis. Un ejemplo que Irma empleaba, me quiero matar porque se me murió el perrito, intentaba mostrar cómo una circunstancia en apariencia ínfima podía desencadenar una crisis.

Rosita, una voluntaria que vi en sólo una ocasión en la sede del CAS me dijo, cuando estaba iniciando mi trabajo de campo, que en esas pequeñas cosas los consultantes se estaban jugando la vida. ${ }^{18}$

...ellos en esa situaciones se han sentido tan desvalidos, a lo mejor por pequeñeces, pero en ese momento era magnificados, para ellos se jugaba la vida, lo que para uno podría ser una pequeñez, ellos se estaban jugando la vida. Entonces esa, el saber escuchar, sin juzgar, el saber ayudarlo a escarbar, como una linterna que le hace ver otras cosas que no está viendo. (Entrevista con Rosita, 2 de junio de 2015)

Las voluntarias tenían como premisa no juzgar los motivos de las crisis, es decir, no emitir juicios valorativos respecto de las situaciones que podían desencadenar ese estado. Lo que implicaba que cualquier situación merecía el mismo tratamiento en las técnicas de las voluntarias. Al mismo tiempo implicaba considerar que las crisis trascendía las clases sociales, por lo que las voluntarias debían estar dispuestas a encontrarse con causas bien diversos en los llamados.

En las actuaciones de las voluntarias del CAS, la crisis se configuraba como el eje articulador de las prácticas. Caso diferente relata Martins (2015) en su investigación desarrollada en el Brasil, donde la moralidad que orientaba las prácticas se fundaba en la idea de "la soledad" que caracterizaría la vida en la ciudad contemporánea. Las

18 Rosita tenía 73 años cuando la conocí. Había ejercido la docencia y llegando a ser directora de una escuela. Hacía más de 20 años que participaba como voluntaria del CAS, donde había ingresado luego de escuchar sobre la institución en la radio. Cuando le pregunté cómo era un día en el CAS me dijo: para mí el jueves es un... viste que el domingo es distinto, el jueves para mí el distinto, porque no hago nada de lo que yo habitualmente hago, es una vacación de lo habitual, y una novedad no habitual, pero que se hace habito al ser sistemáticamente todos los jueves. 


\section{Nanduty}

ISSN:2317-8590

técnicas de las voluntarias se orientaban hacia una resolución favorable de los estados de crisis, suponiendo que, en última instancia, el suicidio era una manera irreversible de resolución de un estado que sería transitorio. De modo que sus prácticas, en este universo, implicaban lo que se llamaba Intervención en crisis.

La idea de que la resolución inadecuada de una crisis, con el enorme costo personal, físico y psicológico, puede conducir a un deterioro psíquico a largo plazo, es, según Slaikeu, una de las piedras angulares de la Teoría de la Crisis: "En tanto que las crisis se caracterizan por un colapso en la capacidad de resolver problemas, la ayuda externa es fundamental para determinar cómo sortearán los individuos estos puntos de cambio". De esta manera Slaikeu define la Intervención en Crisis como: “...un proceso de ayuda dirigido a auxiliar a una persona o familia a sobrevivir un efecto traumático, de modo que la probabilidad de efectos debilitantes (estigmas emocionales, daño físico) se minimiza, y la probabilidad de crecimiento (nuevas habilidades, perspectivas y opciones de vida) se maximiza...". (Documento Crisis-clase. Resaltado en el original)

La cuestión que señala el autor referenciado, de disminuir los efectos debilitantes y aumentar las posibilidades de crecimiento, era condensado por las voluntarias en la idea de que la crisis podía pensarse como amenaza y oportunidad. Dependiendo del afrontamiento que se le dé, una crisis podría convertirse en una amenaza, poniendo en riesgo la integridad del sujeto, o en una oportunidad de aprendizaje o de crecimiento personal. Para conjugar esta idea, las voluntarias en ocasiones recurrían al vocablo chino para la palabra crisis: Weiji, compuesta por los ideogramas de peligro y oportunidad.

El objetivo primario del asistente es, entonces, auxiliar a la persona a dar pasos concretos hacia el enfrentamiento con la crisis, lo cual implica el control de los sentimientos o los componentes subjetivos de la situación, y comenzar el proceso de solución del problema. (Documento Crisis-clase. Resaltado mío.)

Las actuaciones de las voluntarias correspondían al momento de los primeros 


\section{Nanduty}

ISSN:2317-8590

auxilios psicológicos o intervención de primera instancia. ${ }^{19}$ Las actuaciones de las voluntarias no pretendían necesariamente la resolución inmediata de las crisis sino orientar como una linterna o como un faro que ayudase a sortear aguas turbulentas. Gustavo Blázquez y Cecilia Castro (2015) proponen, a partir de sus trabajos etnográficos en fiestas de música electrónica y cumpleaños infantiles, considerar el trabajo de los animadores, VJs y DJs como "gestión de emociones". Gestionar las emociones implicaría una serie de acciones orientadas a organizar tiempos y lugares con el fin de propiciar ciertos estados anímicos y/o evitar otros. De esta forma se incitarían ciertos comportamientos al hacerlos deseables.

La gestión de emociones implicaría administrar

"los flujos afectivos, determinar los ritmos del humor, establecer los momentos y los espacios para las erupciones emotivas alegres o desdichadas, la calma, el relax; [...] consistiría en 'calentar' o 'enfriar' (Schechner, 2000) una determinada performance social por medio de la manipulación del escenario y del sensorium". (Blázquez \& Castro 2015:3)

Interpreto que, en el llamado una cuestión central era "enfriar" al consultante, que salga del estado de crisis emocional en el que se encontraba al momento de marcar 135 en sus teléfonos. Irma decía que podía escuchar cuando al final hasta casi sonríen, porque uno siente la sonrisa. Escuchar eso la gratificaba: misión cumplida.

Blázquez \& Castro considerarán el oficio de animador de fiestas, VJ y $\mathrm{Dj}$ como un oficio de "gestor". En la trasmisión del oficio de telefonista a las aspirantes, pude observar cómo aprendían maniobras orientadas a bajar la ansiedad de los consultantes, tranquilizarlos, ordenar sus ideas y orientarlos en la búsqueda de soluciones. La telefonía en la asistencia al suicida implicaba la manipulación de los sonidos, tiempos,

19 Slaikeu (1996) define cinco componentes en los Primeros Auxilios Psicológicos, que resumen un modelo básico de resolución de problemas: 1.- Realizar un contacto psicológico; 2.- Analizar las dimensiones del problema; 3.- Sondear las posibles soluciones; 4.- Asistir a la ejecución de pasos concretos; 5.- Hacer seguimiento para verificar el progreso. 


\section{Nanduty}

ISSN:2317-8590

palabras y los silencios con el fin de "enfriar" el estado emocional de los consultantes intentando, al mismo tiempo, ordenar sus pensamientos. Enfriando las pulsiones emocionales de los consultantes, también intentaban orientarlos en la búsqueda de soluciones a largo plazo, generalmente un tratamiento psicológico o psiquiátrico. En cierta medida, entiendo que las técnicas de las voluntarias tenían una faceta pedagógica, ya que las gestiones que encaraban con los consultantes podrían ser replicadas por ellos mismos en el futuro. Propiciando una auto-gestión más 'templada' de las emociones y de las crisis vitales que no se oriente a una solución drástica o terminante como lo era el suicidio u otras conductas autodestructivas.

Aquí la propuesta eliasiana aporta a la comprensión de estos sentidos en torno a las conductas autodestructivas como violencia. En El proceso de la civilización, Elías (1998) describe el largo proceso por el cual "el conjunto de emociones de los seres humanos va cambiando lentamente en la dirección de un control emotivo más fuerte y más proporcionado" (Elías 1998:14). Un proceso por el cual los umbrales de violencia en las relaciones interpersonales van disminuyendo a medida que crece el monopolio de la violencia legítima por parte de los estados. Así, mientras éstos controlan teóricamente- cada vez más las expresiones violentas sociales e individuales, las personas desarrollan un conjunto de coacciones en pos de un autocontrol de las pulsiones y emociones poderosas en un proceso a la vez "sociogenético" y "psicogenético". El "contrato civilizatorio" implica una reducción de las prácticas violentas y su monopolio por parte del Estado, o reservadas a ciertos espacios específicos y controlados. Por lo que el ejercicio de la violencia, si no es en esas condiciones, es sancionado, mal visto, ilegítimo, se convierte en un tabú.

El proceso de pacificación de las relaciones interpersonales es tal, que su surgimiento en el contexto de nuestras sociedades desata reacciones de pavor, sorpresa, incomprensión o temor. Como consecuencia de esto, según Elías (2009), suele 


\section{Nanduty}

ISSN:2317-8590

preocuparnos más qué hace posible el acto agresivo; frente a lo cual propone que una pregunta más pertinente que deberíamos hacer es "por qué no hay más" violencia. La interpretación del suicidio (o prácticas autolesivas) como violencia autodirigida, implica un supuesto por el cual dichas conductas estarían atentando contra el proceso civilizatorio. Atentando en el sentido que suponen una tramitación de las emociones que no responde al autocontrol correspondiente a la pacificación propuesta por Elías. A raíz de esto, no se trataría solamente de que el suicidio como muerte "elegida" quebrante la norma social respecto de las mejores formas de morir (Aries 2000). No es suficiente considerar que el suicidio se signifique como una muerte poco legítima, o como un escape último a diversas formas del poder (Rodrigues 1983, Foucault 1996b [1975]). Sería, en el conjunto de saberes articulados por las voluntarias, una forma de resolver conflictos de diversa índole por una vía "violenta". Una forma que confronta las modalidades esperables de tramitación de las emociones, del autocontrol de sí y de las pulsiones violentas configuradas a lo largo del "proceso civilizatorio".

\section{PALABRAS FINALES}

En el principio de este trabajo, invocando un clásico como "El Suicidio", de Durkheim, procuré señalar cómo las preocupaciones analíticas de la sociología eran también preocupaciones gubernamentales. En el marco de configuración del "gobierno" como forma de ejercicio de poder privilegiado, la sociología intentaba establecerse como una disciplina productora de saberes eficaces para la gestión de individuos y poblaciones. Presenté el CAS de la ciudad de Córdoba y su particular proceso de conformación. Además, indagué cuáles eran las preocupaciones analíticas que orientaron mi anterior pesquisa etnográfica y la escritura de este trabajo. Finalmente me detuve en las preocupaciones gubernamentales de las voluntarias respecto del suicidio y cómo se configuraban en torno de los saberes respecto de la noción de Crisis. 


\section{Nanduty}

ISSN:2317-8590

Para lograr apreciar las particularidades de las actuaciones de las voluntarias fue fundamental considerarlas como conformando una "tradición" administrativa, en el sentido que formuló Antonio C. de Souza Lima. El autor denominó

"tradición de conocimiento para la gestión colonial a un conjunto de saberes/haceres, incorporados y reproducidos en patrones habituales de interacción, objetivados en codificaciones, elementos materiales de cultura (arquitectura, indumentaria, etc.) e incorporados en etiquetas, disposiciones corporales, gestos estereotipados. Descubrir y diseminar informaciones, someter y definir, clasificar y jerarquizar, aglutinar y localizar los pueblos conquistados y los espacios habitados por ellos son operaciones desarrolladas por lo que llamo saberes de gestión y por los poderes por los cuales se hacen y generan." (2013: 808 La traducción me pertenece $)^{20}$

Esta noción fue desarrollada por el antropólogo brasilero en sus investigaciones acerca del accionar de la Fundación Nacional del Indio del Brasil (FUNAI) para indagar en torno de la formación y el desempeño de especialistas de dicha agencia. Podríamos pensar que este conjunto de saberes/haceres de las voluntarias también se orientaban a la (co)producción de categorías de sujetos -consultantes, adolescentes en riesgo- y a la administración de sus relaciones entre sí, con sus sentimientos y con otros saberes/haceres provenientes del mundo PSI.

Souza Lima explica que estos saberes orientados a la gestión, no tienen

20 "Estou chamando de tradição de conhecimento para gestão colonial um conjunto de saberes/fazeres, quer incorporados e reproduzidos em padrões costumeiros de interação, quer objetivados em codificações,elementos materiais de cultura (arquitetura, indumentária etc.) e incorporados em etiquetas, disposições corporais, gestos estereotipados. Descobrir e disseminar informações, submeter e definir, classificar e hierarquizar, aglutinar e localizar os povos conquistados e os espaços por eles habitados são operações desenvolvidas pelo que chamo de saberes de gestão e pelos poderes pelos quais se fazem e geram." (Souza Lima 2013: 808) 


\section{Nanduty}

ISSN:2317-8590

necesariamente su origen en agencias estatales, aunque pueden cristalizarse allí. Además propone que "los especialistas en la gestión cotidiana de las formas de gobierno enquistadas en los modos de dominación son productores y transmisores de saberes que tienen una historia propia" (Souza Lima 2013: 807. Traducción propia). ${ }^{21}$ Considerando entonces a las voluntarias del CAS como especialistas en estas formas de gestión de vidas y emociones, es posible reivindicar las particularidades del desarrollo local de sus prácticas. Y al mismo tiempo habilita la posibilidad de desarrollar una indagación genealógica de los saberes/haceres articulados en sus actuaciones en relación con tradiciones de gestión de poblaciones de más larga data.

Las actuaciones en pos de la evitación del suicidio se centraban en la vida, que era donde podían obrar. Que la vida pueda más no era solamente una oposición con 'la muerte', no alcanzaba con considerar que implicaba un triunfo de la vida sobre la muerte. A partir de la pesquisa fue posible vislumbrar que siempre se trataba de la vida. Que ciertas formas de vivir podían más que otras. Las "gestiones" de las voluntarias se orientaban a "gestar" esas nuevas y mejores formas de vivir, constituyéndose sus prácticas en "prácticas de gobierno".

En el ámbito argentino, varias investigaciones han señalado algunos elementos que caracterizarían una gubernamentalidad neoliberal y su desarrollo histórico. Por ejemplo, Murillo (2011) plantea una centralidad del sujeto como objeto del arte neoliberal de gobierno, particularmente modelando su deseo. Así, la cuestión social se gestionaría desde el individuo, lo que al mismo tiempo implicaría una conducción de las propias conductas. En la gubernamentalidad neoliberal, el gobierno de los sujetos sería

21 "De maneira mais abrangente, isto significa reconhecer que os especialistas na gestão cotidiana das formas de governo encasteladas nos modos de dominação são produtores e transmissores de saberes que têm uma história própria, objeto para a investigação genealógica e para o estudo antropológico das formas de poder nos seus desdobramentos no tempo, estudos que sejam capazes também de dialogar com a (macro)sociologia histórica.” (Souza Lima 2013: 807) 


\section{Nanduty}

ISSN:2317-8590

inescindible del autogobierno, de un cuidado de sí muchas veces orientado al triunfo en diversas competencias. Ya en Córdoba siguen en esta línea los trabajos etnográficos de Landa \& Marengo (2011) o de Landa, Blázquez \& Castro (2019) que indagan respecto de la gestión empresarial de cuerpos y emociones, en el primer caso, y sobre el emprendedurismo y la "actitud" entre trabajadores del mundo del fitness y del entretenimiento infantil, en el segundo. Estos trabajos, por solo mencionar algunos desarrollos locales, permiten reflexionar sobre cómo las particularidades de la tradición administrativa aquí analizada, participan de un horizonte mayor de prácticas gubernamentales y de la modulación neoliberal de subjetividades. Prácticas caracterizadas por la centralidad del individuo, en este caso de la individualización de la responsabilidad de una tramitación pacificada de crisis emocionales bastante permeada por saberes PSI. Como desarrollé, la producción social del suicido como un problema gubernamental en Córdoba, con saberes, agentes y agencias orientadas a su evitación, implicaba técnicas que eran individualizadoras en su concepción. Las técnicas del CAS, tanto las telefónicas y como las estrategias preventivas, eran aparentemente efímeras en el tiempo (los minutos de una llamada, o el par de horas de los talleres), pero se orientaban a que sus repercusiones se prolongaran mediante la autogestión de los consultantes, estudiantes, padres y/o personal de las escuelas destinatarias de sus actuaciones.

Aquí, como en el trabajo en Córdoba de Landa, Blázquez y Castro (2019) estas prácticas, con algunas de las características señaladas para la gubernamentalidad neoliberal, eran desarrolladas por agentes que strictu sensu no participaban de la pretendida 'esfera' estatal. En referencia a la relación entre "estado" y "gobierno", Foucault planteó que éste "es a la vez exterior e interior a [aquel], porque son las técnicas de gobierno las que permiten definir en todo momento lo que debe y no debe 


\section{Nanduty}

ISSN:2317-8590

estar en la órbita del Estado, lo que es público y lo que es privado, lo que es estatal y lo que no lo es" (2004: 137). Que la vida pudiera más no se reducía a una oposición con "la muerte". Prácticas de gobierno establecidas en torno del suicidio que estaban configurando un campo de acción, de responsabilidad, de pertinencia que en el último tiempo había comenzado a cristalizarse en prácticas estatalizadas como ser la sanción a nivel nacional de una Ley de Prevención del Suicidio (Ley 27.130) en 2015 y la adhesión de la provincia de Córdoba en $2016 .^{22} 30$ años de trayectoria de Centros de asistencia al Suicida a lo largo del país, permiten reflexionar respecto de cómo el ejercicio de prácticas gubernamentales por parte de agentes de ONGs y/o asociaciones civiles, habilitan la extensión del estado creando prácticas y problemáticas de devienen de interés público y objeto de injerencia estatal y que pueden cristalizarse en la promulgación de leyes y la formulación de políticas públicas.

\section{REFERENCIAS BIBLIOGRÁFICAS}

BLÁZQUEZ, Gustavo \& CASTRO, Cecilia. (2015). "¡Los quiero bien arriba! Gestión de emociones en eventos festivos". Ponencia presentada en las XI Jornadas de Sociología. Coordenadas contemporáneas de la sociología: tiempos, cuerpos, saberes. Facultad de Ciencias Sociales, UBA. MESA 14: Sociología de los cuerpos y las emociones.

http://jornadasdesociologia2015.sociales.uba.ar/altaponencia/acciones2=ver\&id_mesa= 29\&id_ponencia=209

CASULLO, María Martina. (1994). "Comportamiento suicida adolescente. Epidemiología y prevención". En QUIROGA, S. (org). Patologías de la autodestrucción en la adolescencia, Buenos Aires: Kargieman. p. 68 - 92

(1997). "Riesgo suicida en estudiantes universitarios. Epidemiología y prevención". Desarrollos en Psiquiatría Argentina, 2,

22 Adriana Vianna (1997) desarrolla cómo las prácticas policiales se cristalizaron en el Código de Menores de 1927, llamando la atención sobre cómo muchas veces las leyes y normativas no fundan prácticas, y que en algunos casos las lógicas administrativas son previas. 


\section{Nanduty}

ISSN:2317-8590

$4: 33-38$.

(1998). Adolescentes en riesgo: identificación y

orientación psicológica. Buenos Aires: Paidós.

(2006b). "El capital psíquico. Aportes de la

Psicología Positiva". Psicodebate. Psicología, Cultura y Sociedad Nº6: 59-72.

. (2009). Prácticas de Psicología Positiva. Buenos

Aires: Lugar Editorial.

y FERNÁNDEZ LIPORACE, María Mercedes.

(2006). "Validación factorial de una escala para evaluar riesgo suicida". Revista Iberoamericana de Diagnóstico y Evaluación - e avaliação Psicológica 1(21): 9-22.

; FERNÁNDEZ LIPORACE, María Mercedes y

BONALDI, Pablo Daniel. (2000). Comportamientos suicidas en la adolescencia. Morir antes de la muerte. Buenos Aires: Lugar Editorial.

DURKHEIM, Émile. (1985). Las reglas del método sociológico. Buenos Aires: La Pléyade. (1998) [1897]. El suicidio. Madrid: Akal ediciones.

EDWARDS Catherine y OSBORNE Thomas. 2005. "Scenographies of suicide: An introduction". Economy and Society 34(2): 173-177.

ELIAS, Norbert. (1987). El proceso de la civilización. Investigaciones sociogenéticas y psicogenéticas. Madrid: FCE.

. (2009). Los Alemanes. Argentina: Editorial Nueva Trilce.

ESQUIVEL, Valeria; FAUR, Eleonor y JELIN, Elizabeth. (2009). Hacia la conceptualización del cuidado. Buenos Aires: IDES-UNICEF-UNFPA.

FOUCAULT, Michel. (1988). "El sujeto y el poder". Revista Mexicana de Sociología, vol. 50 no3 (jul-set): 3-20.

(1996). "Omnes et singulatim: hacia una crítica de la «razón política»". La vida de los hombres infames. Editorial Altamira. La Plata.

(1996b) [1975]. Genealogía del racismo. La plata Ed. Altamira.

. (2006) [1978]. Seguridad, territorio y población. Curso en el

Collège de France (1977-1978). Buenos Aires: Fondo de cultura económica.

Barcelona: Paidós. p. 857-859.

(2010). "Un placer tan sencillo". En Obras esenciales.

. (2013) [1976]. Historia de la sexualidad I. Voluntad de

Saber. Buenos Aires: Siglo XXI editores.

GEERTZ, Clifford. (1990). La interpretación de las culturas. Barcelona: Gedisa.

GIDDENS, Anthony. (1965). "The suicide problem in French sociology". The British Journal of Sociology 16(1): 3-18.

LANDA, M.I., BLAZQUEZ, G. \& CASTRO, C. (2019). "Emprender como estilo de 


\section{Nanduty}

ISSN:2317-8590

vida. La actitud en las dinámicas laborales de trabajadores del fitness y el entretenimiento infantil (Córdoba, Argentina)". Debats. Revista de cultura, poder y sociedad, 133(1), pp. 27-45.

LANDA, M. y MARENGO, L. (2011). "El cuerpo del trabajo en el capitalismo flexible: lógicas empresariales de gestión de energías y emociones". Cuadernos de Relaciones Laborales, 29(1), pp. 177-199.

LENOIR, Remi. (1993). "Objeto sociológico y problema social". In CHAMPAGNE, P; LENOIR, R; MERLLIÉ, D; PINTO, L. (org). Iniciación a la practica sociológica, editado por. México, Siglo XXI Editores. p.57-80.

LIMA, Antónia Pedroso de. (2016). "O cuidado como elemento de sustentabilidade em situações de crise. Portugal entre o Estado providência e as relações interpessoais". Cadernos Pagu janeiro-abril de 2016:79-105.

MARTINS, Isis Ribeiro. (2015). “A linha da vida”: escuta e gestão do sofrimento em serviços telefônicos de apoio emocional. Tesis Doctoral. Rio de Janeiro: UFRJ/Museu Nacional/PPGAS.

(2016). "Moralidades e atos de fala em serviços de apoio emocional: Modalidades laicas da confissão e do testemunho?" Religião e Sociedade, Rio de Janeiro, 36(2): 19-43

MURILLO, Susana. (2011). "Estado, sociedad civil y gubernamentalidad neoliberal". Entramados y Perspectivas. Revista de la carrera de Sociología. Buenos Aires. 1(1): 91-108.

PAUTASSI, Laura y ZIBECCHI, Carla. (2013). Las fronteras del cuidado. Agenda, derechos e infraestructura. Buenos Aires: Biblos.

RODRIGUES, José Carlos. (1983). Tabu da Morte. Achiamé, Rio de Janeiro.

ROMERO, M.A. y GONNET, J.P. (2013). "Un diálogo entre Durkheim y Foucault a propósito del suicidio" en Revista Mexicana de Sociología 75, núm. 4 (octubrediciembre, 2013): 589-616.

ROMERO, Santiago Manuel. (2018). La vida puede más. Formas de asistencia y prevención del suicidio de un Centro de Asistencia al Suicida de la ciudad de Córdoba, Argentina. Trabajo Final de Licenciatura en Antropología, Universidad Nacional de Córdoba.

SCHECHNER, Richard. (2000). Performance. Teoría \& Prácticas Interculturales. Buenos Aires: Libros del Rojas. Universidad de Buenos Aires.

SLAIKEU, Karl. (1996). Intervención en crisis: manual para practica e investigación. Buenos Aires. Manual Moderno.

SOUZA LIMA, Antonio Carlos de. (2013). "O exercício da tutela sobre os povos indígenas: considerações para o entendimento das políticas indigenistas no Brasil contemporâneo". Revista de Antropologia, Sao Paulo, USP v. 55, n. 2: 781-832. 


\section{Nanduty}

ISSN:2317-8590

TIERNEY, Thomas F. 2010. "The governmentality of suicide: Peuchet, Marx, Durkheim, and Foucault". Journal of Classical Sociology 10(4): 357-389.

VIANNA, Adriana de Resende Barreto. (1997). O mal que se adivinha. Polícia de minoridade no o Rio de Janeiro, 1910-1920. Rio de Janeiro: Arquivo Nacional.

ZIBECCHI, Carla. (2014). "El trabajo de cuidado. Invisibilidad e inequidades de género en la Argentina actual". Revista Científica Equipo Federal del Trabajo. Equipo Federal del Trabajo - Facultad de Ciencias Sociales de la Universidad Nacional de Lomas de Zamora. 